dent of climate and season, and furnishing him with a power of producing new varieties.

Before electro-horticulture can be entertained as a practical process it would be necessary hovever to prove its cost, and my experiments of last winter were in part directed towards that object. Where water-power is available, the electric light can be produced at an extremely moderate cost, comprising carb $m$ electroles, and wear and tear of and interest upon apparatus and machinery employed, which experience elsewhere has already shown to amount to $6 d$. per hour for a light of 5000 candles. The personal current attention requisite in that case consists simply in replacing the carbon electroles every six or eight hours, which can be done without appreciable expense by the under-gardener in charge of the fires of the greenhouses.

In my case no natural source of power was available, and a steam-engine had to be reso:ted to. The engine of 6 normal horse-power which I employ to work the two electrie lights of 5000 endle-power each, consumes 56 lbs. of coal per hour (the engine being of the ordinary high-pressure type), which, taken at 20 s. a ton, would amount to $6 . l$. or to $3 d$. per light of 5000 candles. But against this expenditure has to be placed the saving of fuel effected in suppressing the stoves for heating the greenhouses, the anount of which I have not been able to ascertain accurately, but it may safely be taken at two-thirds of the cost of coal for the engine, thus reducing the cost of the fuel per light to $I d$. per hour; the total cost per light of 5000 candles will thus amount to $6 d,+\mathbf{I} d=7 d$. per hour.

This calculation would hold good if the electric light and enzine power were required during say twelve hours pir diem, but inasmuch as the light is not required during the daytime, and the firing of the boiler has nevertheless to be kept up in order to supply heat to the greenhouses, it appears that during the daytime an amount of motive-power is lost equal to that employed during the night.

In order to utilise this power I have devised means of working the dynamo-machine also during the daytime, and of transmitting the electric energy thus protuced by means of wires to different points of the farm, where such operations as chaff-cutting, swede-slicing, timber-sawing, and water-pumping have to be performed.

These objects are accomplished by means of small dynamomachines plucel at the points where power is required for the-e various purposes, and which are in metallic connection with the current-generating dynamo-machine near the en gine. The connecting wires employed consist each of a naked strand of copper wire supported on wooden poles or on trees without the use of insulato:s, whilst the return-circuit is effected through the parkrailing or wire fencing of the place, which is connected with both transmitting and working machines by means of short pieces of connecting wire. In order to insure the metallic continuity of the wire fencing, care bas to be taken wherever there are gates to solder a piece of wire, buried below the gate, to the wire fencing on either side.

As regard; pumping the water, a 3-horse-power steam-engine was originally used, working two force-pumps of $3 \frac{1}{2}$-inch diameter, making thirty-six double strokes per minute. The same pumps are still employed, being now worked by a dynamomachine weighing $4 \mathrm{cwt}$. When the cisterns at the $\mathrm{h}$ ouse, the gardens, and the farm require filling, the pumps are started by simply turning the commutator at the engine station, and in like manner the mechanical operations of the farm already referred $\left.t_{1}\right)$ are accomplished by one and the same prime mover.

It would be difficult in this in tance to state accurately the percentage of power actually received at the distant station, but in trying the same machines under similar circumstances of resistance with the aid of dynamometers, as much as 60 per cent. has been realised.

In conclusion, I have plea ure to state that the working of the eiectric light and transmission of power for the various operations just named are entirely under the charge of my headgardener, Mr. Buchanan, assisted by the ordinary staff of under-gardeners and field-labourers, who probably before never heard of the power of electricity.

Electric transmision of power may eventually be applied also to thrashing, reaping, and ploughing. These objects are at the present time accomplished to a large extent by means of portable steam-engines, a class of engine which has attained a high degree of perfection; but the electric motor presents the great advantage of lightness, its weight per horse-power being only 2 cwt., whilst the weight of a portable engine with its boiler filled with water may be taken at 15 cwt. per horse-power. Mureover, the portable engine requires a continuous supply of water and fuel, and involves skilled labour in the field, whils the electrical engine receives its food thruugh the wire (or a light rail upon which it may be made to $m$ ove about) from the central station, where power can be produced at a cheaper rate of expenditure for fuel and labour than in the field. The use of secondary batteries may also be resorted to with advantage to store electrica! energy when it cannot be utilised.

In thus acconplishing the work of a farm from a centralpower station, considerable savings of plant and labour may be effected ; the engine-power will be chiefly required for day work, and its night work for the purposes of electro-horticulture will be a secondary utilisation of the establishment, involvin; little extra expense. At the same time the means are provided of lighting the hall and shrubberies in the $m$ st perfect manner, and of producing effec's in landscape gardeni!ng that are strikingly beautiful.

\section{THE ELECTRICAL DISCHARGE, ITS FORMS AND ITS FUNCTIONS ${ }^{1}$}

\section{II.}

A MONG the various circunstances which combine to determine the character of the discharge, one of the most important is the size of the negative terminal. And in this respect, as well as in others, the negative differs fundamentally from the positive. If the negative be small, not so much in comparison with the positive as in absolute magnitude, and perhaps al $o$ in reference to the diameter of the tube, the tube will offer great "resistance," as it is termed, to the passage of the discharge. On the other hand, if the negative be large, the discharge passes with comparative ease. In the first case, even when the discharge passes, strix are formed only with difficulty, if at all ; in the second they are readily formed. This may ea-ily be shown by using a tube with one small and one large terminal, which can be used alternately as positive and as negative; or by a tube having a negative terminal of variable length.

The same dependence of striation upon the size of the negative may be shown in the case of a tube with a negative terminal of barely sufficient size. In this case, if the tube be touched by the hand (an operation which, as will be hereafter explained, is equivalent to enlarging the negative), striæ will be brought out clear and distinct, while without this arsistance they appear only in a confused and irregular manner.

Other characteristic features of the negative terminal would deserve our attention if time permitted. Thus, the well-known phenomena of the so-called "Holtz tube" (or tube divided into compartments by diaphragms furnished with narrow pipes leading from one compartme it to the next, and all pointed in one direction), show that a small aperture will serve as a negative, but not as a positive terminal. This property has been generalised by $\mathrm{G}$ )ldstein, who, using as a negative terminal a cylinder of non-conducting substance pierced with fine holes, reproduces all the phenomena appertaining to an ordinary metallic negative.

And, even apart from the phenomena of vacuum tubes, it would not be difficult to adduce instance: showing the importance of the size of the negative terminal in electrical discharges generally. Of these I will now mention only the latest. In making some modifications of Planté's battery M. de Pozzer has found that, if the negative electrode be made of a plate of lead of half a millimetre in thickness, and the positive of one of twothirds of a millimetre, but the former double the size of the latter, great advantage arises from the greater size of the negative. The discharge from a battery having a negative double as large as the positive lasted, on an average of several experiments, for an hour; while that from a battery, in which the sizes of the electrode: were rcversed, lasted only half an hour. The effect of a battery with electrodes of equal size appears to have been internediate to that of the two others.

From these phenomena, and especially from those of the moving terminal, as well as from other considerations, it appears that the general confi, uration of the discharge is mainly determined at the negative terminal.

In order, however, to experiment with any hope of progress

${ }^{I}$ A Lecture delivered before the British Association at York on September 5, 188r, by William Spottiswoode, D.C.L., LL.D., President of the Royal Society. Continued from p. 55 r. 
in our knowledge of the nature and modus operandi of the discharge, we ought to be in a position to modify the discharge, so as to compare it under different circumstances. The methods hitherto usually employed for this object have been an alteration in the gas used, an alteration of the pressure, and a diversity of figure in the tube or in it; terminals. Of the general character of the changes due to such alterations we have already seen something. But, besides these alterations, which are of a structural or instrumental character, it is also desirable to operate on a disch arge actually in transitu. One of the methods, in fact the only one, employed until lately is that of the magnet, which was used as long ago as the time of Grove, Pliicker, and other early experimenters. It is well known that a magnet will displace a movable conductor when carrying a current, according to laws established by Ampère. The same is true, in general terms, with respect to a discharge traversing an exhausted tube.

Thu: in the tube now before you, you will see that when one pole of a magnet is presented to the tube, the discharge is thrown to one side of the tube; and when the other pole is so presented the discharge is thrown to the other side. These two main features, however, very inadequately describe the actions of a magnet, which in fact operates separately not only upon each stria as a unit, but even upon the various parts of a stria in such a way as to deform it a; well as to displace it. But they involve the main characteristics of the magnetic action, and must suffice on the present occasion to show that in the magnet we have a powerful instrument for examiling the properties and functions of the discharge, even (if the term may be permitted) in the living specimen.

The other principal mode of operating on the discharge consists in reducing it to what has,been called the "sensitive state", i.e. to a state in which the position of the luminosity is affected by the approach of a conductor to the tube. For the details of an experimental investigation into the phenomena of this state, and a discussion of the conclusions that may be drawn therefrom, the reader is referred to the Philosophical Transactions of 1879 and $188 \mathrm{o}$. But the following remarks may serve to convey some notion of the method and its issues. Sensitiveness is produced by breaking the circuit with a short interval of air, or, as it is usually described, by interposing an "air-spark" in one branch of the circuit, viz. either that leading from the positive, or in that leading from the neyative, terminal of the machine to the tube, or by otherwise rendering the discharge intermittent. The effect of this is to discharge the electricity discontinuously, so that from time to time there passes into the tube a comparatively large quantity of electricity at a higher tension than would otherwise be the case. By this means the gas in the interior of the tube, or perhaps the interior surface of the tube itself, becomes mom entarily charged with electricity, thus creating an electric tension, which may be discharged or "relieved" by a displace. ment in the electricity on a conductor brought near, or in contact with, the tube. This causes or permits a discharge from the interior of the tube itself of the electricity of an opposite kind to that with which the tube itself is charged. If the air spark is on the positive side the charge on the tube is positive, and the relief negative, and vice versâ. From this it follows, as might have been expected, that the effect of the relief on the visible discharge is different in the two cases. In each case the part of the inner surface of the tube nearest to the conductor acts as a quasi terminal. As a general rule, with a positive air-spark, the relief, being negative, tends to produce a dark space, and thereby gives the appearance of a repulsion of the luminous column. With a negative air-spark the relief, being positive, tends to produce a stria ; it thereby cau es luminosity, and gives an appearance of attraction of the luminous column.

The appearance of the luminous column when produced under the action of an air-spark is usually amorpbous or unstratified, although this is not always the case. In the case of a positive air-spark the column is more or less constricted and confined to the central part of the tube ; in the case of a negative air-spark it is more diffused, and usually fills the whole diameter of the tube. This is doubtless due to a gradual discharge from the sides of the tube, and is in accordance with what has been said above.

Under suitable circumstances this relief discharge may be made to bring out artificial striæ from an amorphous discharge, the position of the striæ depending upon the character of the airspark used. The positions occupied by strix in the one case will be occupied by dark spaces in the other, and vice versâ.

The facts here adduced, together with many others based upon a long series of experiments, all tend to the conclusion that, whatever the number or form of the striæ in a stratified column, each stria is to be regarded as a physical unit, and that in each unit we have represented all the elements of a complete discharge. The form of each stria is in every case determined by that of its immediate predecessor, reckoned from the negative end. This may be verified, among other ways, by observing the form of the successive strix when distorted by the influence of a magnetic field. The same research has further established the fact that the regative glow and the haze behind it, which terminates in what is usually known as the negative dark space, is a stria turnet as it were inside out by the influence, the shape, and the character of the negative terminal. From the mode in which this stria is connected with the negative terminal it has been called the " anchored stria,"

The relief effects, may, however, be produced equally well by connecting a point on the surface of the tube with the opposite, or non-air-spark terminal, instead of with earth. By this means we supply a charge of electricity of the opposite name to that with which the tube has been charged, and obtain a result of a similar character to that of ordinary relief.

Alongside of the relief effects above mentioned there is also a system of what we have termed "special effecte," which latter are converse to the former, each to each. These are produced by connecting a point on the outside of the tube with the airspark terminal itself. The special effect with a positive airspark is equivalent to a relief effect with a negative air-spark, and vice versâ.

Lastly, all these effects may be produced by mean: of impulsive discharges to the outside of the tube from an independent source of electricity, such as a second Holtz machine. And, mutatis mutandis, the corresponding effects may be produced by this method even on a non-sensitive discharge. This completes the entire cycle of phenomena due to impulsive action $a b$ extra.

The character of these effects being known once for all, this impulsive action may be used as a test of the nature of a discharge (i.e. whether positive or negative) passing through a given tube. For example, we may experimentally verify in the case of a coil di-charge what might have been anticipated on the principles now established. Such a discharge is in fact equally intermittent from both ends. There is no reason why either terminal should be regarded as the air-spark terminal rather than the other. Hence we might expect that the discharge would be positive through about one-half of the tube, and negative through the remainder, with a neutral zone between them. And such proves to be the case. But more than this, if we attach a small conden er to either terminal of the tube, so as to tone down the impulsiveness of the discharge at that end, we can thereby alter the proportions of the positive and the negative parts of the discharge and shift the position of the neutral zone at will.

The distinctive character which it is thus possible to convey to the whole, or to the two parts of one and the same discharge, naturally leads us to examine whether it be not possible entirely to separate one from the other, and to produce what may be called a unipolar discharge. And this in fact may be done; by connecting the one terminal of the tube through an air-spark to one branch of the circuit, and by leaving the other disconnected, we may pr duce a discharge which, having plunged blindly into the tube, and finding no response from the other end, returns upon itself, and finds exit by the way by which it came. The uni polar discharge is essentially intermittent, and therefore sensitive the positive is conical in form and tapering towards its end ; the negative is broad, and, so far as it extends, it fills the entire width of the tube. Lastly, two unipolar discharges of the same name can be produced in the same tube; they repel one another, and each returns like a single one.

From these experiments we conclude that the independence of the discharge from each terminal is so complete that we can at will cause discharges from the two terminals to be equal in intensity but opposite in sign (as in the case of the coil), or of any degree of inequality (as in the case of the coil with a small condenser). Or we can cause the discharge to be from one terminal only, the other terminal acting merely receptively (as in the case of the air-spark discharge); or we can cause the di charge to pass from one terminal only and return to it, the other terminal not taking any part in the discharge ; or, finally, we can make the two terminals pour forth independent discharges of the same name, each of which passes back through the terminal whence it came.

One of the most important consequences which follows from 
these considerations is that the discharges at the two terminals of the tube are so far independent as to be primarily determined each by the conditions at its own terminal, and only in a secondary degree, if at all, by the conditions that exist at the opposite terminal. And since the discharges are not necessarily the same at both terminals, the tube must contain free charges at different time:. A tube is therefore not like a conductor, but is an independent electrical system, holding much the same position as the air-vessel in a forcing-pump. All the electricity that goes into it goes out again, but this is true only when we consider the whole discharge from the beginning to the end, and it may not be true even approximately during a small finite time. This independence of the discharges from the two terminals in the passage of electricity through rarefied gases dissipates the error of seeking analogies in metallic conduction; and shows that any obedience to regular laws as to change of potential as we proceed along the tube, resistance, \&c., must arise from the fact that the effects measured are really average effects over an interval of time very long compared with the duration of the individual discharges.

The importance which attaches to the negative end of the discharge has led experimenters to examine whether the features appertaining to it could not be still further enlarged. And the only thing requisite to carry the experiments to a limit was an instrumental method of improving the vacuum to the degree required. This was furnished by Mr. Crookes' refinements on the Sprengel pump. In a series of most remarkable experiments he has shown, as mentioned above; not only that the striated column may be reduced to zero, but that the anchored stria itself may be so driven back that the blank space in question may be made to occupy the entire length of the tube.

When an exhaustion cuch as that described, or an exhaustion nearly equal to it, has been reached, a phenomenon, previously noticed, but not before made the subject of serious inquiry, presents it elf. Certain parts of the interior surface of the tube become luminous with phosphorescent light. The colour of this light depends on the nature of the glass, and not in any way on the nature of the residual gas within the tube, nor on the substance of which the terminal is made. With German glass the phosphorescent light is green, with English glass it is blue. But the portion of the glass thus rendered luminous depends upon the form and position of the negative terminal. This phenomenon is supposed to be due to the streams of gaseous particles shot off from the neighbourhood of the negative terminal during the discharge. Although there is reason to think that the e streams are an accompaniment rather than an integral part of the discharge, yet the particles would seem to be themselves charged with electricity, inasmuch as their paths are affected by a magnet, just as is a movable conductor carrying a current, or a charged body in rapid motion.

The whole subject of these streams, their power of heating metals and other substances, the shadows cast by bodies interposed in their path, and other properties of them, have been so well and so fully illustrated by Mr. Crookes both in published memoirs and in a lecture before this Association, that it is unnecessary for me now to dwell upon the subject in detail.

Their nature and properties, however, having been thus in the main determined, these streams have proved a valuable auxiliary in an investigation of what have been called the "small time-quantities" involved in the discharge. The discharge is, as has been already shown, a complex phenomenon, the various parts of which, although not entirely separable, may be shown to occupy different periods of time; and the length of these periods may be compared $n$ ith one another, and with other known electrical phenomena. We cannot, it is true, make any abso ute determinations of the time occupied in any of them, but we may still form a table of relative magnitudes of these small time-quantities. And 1 will now endeavour in a few words to give some idea of the nature of these quantities, and of the method whereby they have been measured.

If we take a tube of such high exhaustion as to cause the discharge to become intermittent, or if we use a positive airspark of sufficient length with a tube of fair exhaustion, phosphorescent light, due to molecular streams, will be seen on the inner surface of the glass near the negative terminal. If then a patch of tinfoil, connected with earth, be placed on any other part of the tube, it will cause negative relief discharges to take place from the glass immediately within it, producing phosphorescence on the opposite side of the tube.

If any solid object, such as a piece of wire, should be present in the tube below the point of contact, it will cast a shadow on the phosphorescence, precisely as in Crookes' experiments with the streams from the negative terminal. If there be two points of relief contact, the same object will throw two shadows, in directions conformable with radiations from each. To these, other experiments might be added.

A determination of the precise directions in which these molecular streams issue from a relieving surface is not a very simple problem ; and we must here content ourselves with showing that, in the case of intermittent discharges at least, the streams do not issue normally. If a strip of tinfoil placed along the tube be used as a relieving surface, the phosphorescence takes the form of a sheet wrapped round the tube; if the strip be wrapped round the tub , the phosphorescence takes the form of a sheet laid along the tube. If contact be made with the finger over a finite surface, or by a ring of wire laid close upon the tube, the phosphorescence takes the form, approximately, of the evolute of an ellipse. In all these cases the illumination is somewhat irregular; but the geometrical elements of which the phosphore:cent figure is composed, and the stripes or striations of more intense light, are always formed at right angles to the longer dimension of the contact piece. This being so, suppose that we place on the tube a strip in such a curve that the normal planes to the curve will pass through the tangent at the corresponding point of the image of the curve, i.e. the curve on the opposite side of the tube, each point of which is exactly oppo. site to a point on the tinfoil. In such a case all the striations will lie along the curve formed by the locus of the central patches of phosphorescence, and the result will be a single bright curved line of phosphorescence without any spreading out or striated margin. The curve fulfilling these conditions will be a helix, whose pitch is half a right angle. Experiment confirms the anticipation.

One more step in the study of these molecular streams is necessary for our present purpose, namely, an application to them of the same method which we have used with the electrical discharges themselves; viz. we must examine the effect of an inductive stream produced $a b$ extra upon a direct stream due to the discharge inside the tube. These effects may be described generally as the interference of molecular streams.

If the finger be placed upon a highly exhausted tube through which a discharge with a positive air-spark is passing, the phosphorescence due to the molecular streams from the negative terminal is seen to fade away from the place where the finger rests, and from a region lying thence in the direction of the positive terminal. The effect is that of a shadow over that part of the tube; and as this is produced not by any real intervening object, but by an action from outside, we have termed it a "virtual shadow." The phenomenon is due to a beating down of the streams of molecules coming from the negative terminal, by the transverse streams from the side of the tube immediately within the part touched.

The interference of two molecular streams may be further illustrated by a variety of experiments, and in particular by arranging within the tube a conductor of some recognisable form - say skeleton tetrahedron. If the tube be tonched at a place opposite to this object a shadow of the latter will be formed in the relief phosphorescence; but if the tube be touched also at a point on which the conductor rests, the shadow will be splayed out in a striking manner. This splaying or bulging of the shadow is due to the interference of the molecular streams issuing from the surface of the conductor, which then acts as a quasi negative terminal, with the original relief streams issuing from the first point of contact.

With the help of these properties we are able, by connecting a patch of tinfoil on the tube with earth, or with the negative terminal itself, or with a second patch elsewhere on the tube, to detect the presence or absence of a demand for negative electricity; to localise the main seat of such demand; and even to compare the electrical condition of different parts of the tube at the same time or of the same parts at different times during the very passage of the discharge. In this way we approach the question of the small time-quantities involved in the discharge.

And, in the first place, it must be understood that the whole duration of one of these intermittent discharges is comprised within a period of which the most rapidly revolving mirror has been incompetent to give any account. It may be in the recollection of a few of my audience that when the discharge from my great induction-coil was exhibited at the Royal Institution with tubes on a revolving disk the discharge showed a durational 
character as long as the coil alone was used; but as soon as a Leyden jar was introduced, which was in the main equivalent to an air-spark in a continuous current, the durational character disappeared, and nothing was visible but a bright line, the width of which depended, not upon the duration of the discharge, for no velocity of rotation in any way affected it, but only on the width of the slit through which the discharge in the tube was seen. But, notwithstanding the extreme rapidity with which the discharge is effected, our experiments have already shown that the spark or discharge is a complicated phenomenon, the various parts of which take place in a certain order or sequence of time ; and that in virtue of this sequence we have succeeded, at the various pressures comprised within our range, in affecting and modifying it in transitu. This suggested the idea that, although the subject is surrounded with difficulties, it might still be possible to form some relative estimate, at all events, of the time occupied by the various parts of which the whole phenomenon is composed. And in fulfilment of this the following are some of the conclusions to which we have been led.

The time occupied in the passage of electricity of either name along the tube is greater than that occupied in its passage along an equal length of wire.

This may be shown by connecting metallically a piece of tinfoil near the air-spark terminal with another near the distant terminal; for it is then seen that the former derives as much relief from the latter as if the latter were not on the tube. This shows ( $r$ ) that at the time when the electric disturbance reached the nearer piece of tinfoil the more distant piece was unaffected, and (2) that the disturbance propagated along the wire reached the second piece before the arrival of the same disturbance propagated within the tube.

The negative discharge occupies a period greater than that required by the particles composing the molecular streams to traverse the length of the tube, but comparable with it.

Proofs of this proposition are to be found in the phenomena of virtual shadows, and in other instances of the interference of molecular streams; but, omitting detailed experiments, the general argument on which the above conclusion is based is as follows : If two molecular streams, one issuing with positive relief from the side of the tube, the other coming from the negative terminal, show signs of interference, it is clear that the former of these, which certainly started first, must have continued to flow, at all events, until the arrival of the latter.

The time occupied by the passage of electricity of either name along the tube is incomparably shorter than that occupied by the emission of the molecular streams, or (what is the same thing) the time occupied by the negative discharge.

In support of this conclusion we have time only to mention a single experiment. If two pieces of tinfoil connected by a wire be placed, one near the negative, the other near the positive end of a tube through which a negative discharge with a rather long air-spark is passing, the former will show relief (positive) effects, the latter special (negative) effects; but no phosphorescence will be caused at the latter, however long the air-spark used. When the second patch is lifted off the tube and placed upon another through which no current is passing, phosphorescence is immediately produced. The explanation of this appears to be as follcws : The negative electricity, bursting into the tube, summons all the positive which it can draw from the tinfoil. This is answered so promptly, that the second patch gives up to the first through the medium of the wire all the positive that it can yield, or, which is the same thing, draws off from the first all the negative that it can obtain; and th's is done before the advancing negative reaches the distant patch. But so rapidly does the negative advance, that it reaches the distant patch before the molecular streams have had time to flow from the latter in a sufficient stream to produce phosphorescence ; and it reaches it in time to revoke the supply of positive to the nearer, and to draw back the supply of negative which would bave come to, and with it the molecular streams which would otherwise have flowed from, the further patch. When the second patch is placed on an independent tube, where no such revocation is possible, phosphorescence actually appears, showing that the revocation is no mere supposition, but a real phenomenon.

From the last two laws it follows as a consequence that negative electricity, and therefore also electricity of either name, in the tube outruns the molecular streams.

We may now fairly ask whether the phenomena which we have been studying have any counterpart in the larger operations of nature which are going on around us, and whether the con- clusions to which we have been led afford any explanation of observed facts? Many natural phenomena doubtless fundamen. tally depend upon electricity; how many we hardly yet know. But there are two in particular, namely, lightning and the aurora, which are unquestionably electrical, and whose correspondence with the spark proper, and with the discharge in rarefied gases, respectively has often been noticed. On these I venture to offer a few remarks.

To say that both of these phenomena are dependent on the electrical state of the atmosphere is not saying much; both for other reasons, and especially because we do not know upon what atmospheric electricity itself depends. But it is clear that it is to a knowledge of the distribution of such electricity that we mu.t look for a proximate, as well as an approximate, explanation of the facts.

In a thunder-cloud we have an aggregation of aqueous particles small enough to remain, temporarily at least, suspended in the air. All of these, it would appear, are similarly electrified, ard by their mutual repulsion are restrained from further coalescence. By their presence the ground below the cloud becomes inductively electrified in the opposite sense ; and as soon as the cloud by its motion comes within sparking distance, or by an increase of its charge attains sufficient tension, a spark discharge takes place, which, as we have seen above, is a flash of lightning. A similar action may naturally take place between two clouds oppositely electrified. The electrical tension required for a flash of lightning is of course enormous. It bas been calculated that in order to produce directly from a battery of the most favourable construction a spark of 42 inches, equal to that given from my great induction-coil, from 60,000 to 100,000 cells w ould be necessary; while for a flash of lightning a mile long not less than $3,500,000$ cells would be required.

In some interesting experiments on water flowing from a small orifice in a cistern Lord Rayleigh has found that the breaking of the continuous column into drops is checked by communicating to it a small charge of electricity; but that it is promoted by a large charge. We may imagine with him that something of the same kind tal:es place in the cloud; and thit before the fla: $h$ the aqueous particles are kept apart by mutual repulsion due to their being all highly charged with electricity of the same name ; but that after the flash they are left either without charge or with so slight a charge as to promote their coalescence and their consequent fall in the form of rain. This would be an explanation of the well-known downpour which frequently occurs after a flash of lightning.

There is moreover anotber form of lightning to which the discharge in our vacuum-tubes offers, to say no more of it, considerable analogy, namely, that commonly known as ball lightning. The appearance of ball lightning is described as that of a luminosity or ball of fire moving generally towards the earth, in a direction more or less oblique, and disappearing in most cases before reaching the griund. In some tubes, the exhaustion of which is very moderate, say, having a pressure of several millimetres of mercury, it happens not only that the blocks of light termed entities by Mr. De La Rue are formed, but also that these entities travel along the tube from the immediate neighbourhood of the positive terminal to a finite distance in the direction of the negative, and then disappear. It would seem not unreasonable to sup ose that ball lightning is due to conditions not dissimilar to those of such tubes, namely, to a discharge occurring in the upper regions of the air, at an elevation of perhaps twenty miles, more or less, where the pressure is moderate, that is to say, greater than that under w hich an auroral-like display could take place, and yet less than that which would give rise to a true spark or ordinary flash of lightning. And if further we effect the discharge in the tube by the gradual outpouring of electricity from a charged Leyden battery, or other condenser, through a suitable resistance, or if we use an induction-coil, then the condenser, or coil, will represent the charged cloud, or portion of the atmosphere, from which the phenomenon proceeds; and the analogy.will perhaps be considered sufficiently close to render further observations in proof or disproof of the theory desirable.

Let us now turn to the aurora. Sufficient experiments have been made this evening to show that the discharge in rarefied gases differs from that in gases at higher $\mathrm{F}$ ressures; and that the difference corresponds exactly to that observed between the diffused, gentle, and flickering play of the aurora and the sudden crashing spark of a flash of lightning. It is also abundantly clear that at an elevation of twenty or thirty miles above the 
earth's surface the atmospheric rarefaction must be such as to convert what would be lightning at a lower leve into a discharge similar in the main to that in a vacuum tube.

Furtber, it is an ascertained fact that a difference of electrical condition in different portions of the atmosphere often prevails. We have, therefore, not unfrequently present in regious at moderate elevations, say from twenty to fifty miles, all the conditions necessary for the production of an auroral display.

And $n$ )t only so, but our experiments enable us to determine, at all events approximately, some limits of elevation within which this phenomenon can occur, and thereby to check the very divergent esti nates of those who have observed it. Estimates of the altitude at which the auroral discharge takes place have been mide from simultaneous observations at different points, and these have ranged up to fifty or sixty, and eve $x$ to $28 \mathrm{I}$ miles. But even the lowest of these appears to be improbable. The pressure at which the resistance of air is least is a little less than 4 of a millimetre of mercury; and the corresponding elevation is about thirty-eight miles. A vacuum tuhe meacured by hundred-thousandths of an atmosphere would correspond to an elevation of a little more than eighty-one miles. Through a hydroge ? vacuum at this pressure Mr. De La Rue failed to obtain a discharge with II, 0oo cells; : nd he add : that "it may be assumed that at this height the discharge would be considerably less brilliant than at thirty-eight miles, should such occur."

It seems to be a well-ascertained fact that in high latitude there are fewer thunderstorms and more auroras than in lower latitudes. This fact points to the conclusion that, after a dis'urb ance, the re-distribution of atmospheric electricity is effected by one process or by the other, according to, or rather in consequence of, the meteorological differences between arctic, temperate, and tropical regions. In colder regions, where the air is generally drier, and, consequently, a better insulato: than in warmer, there is less liability to a discharge taking place in the lower and denser strata; that is, there is less liability to lightning. But at higher levels the rarefaction may compensate this, and cause an auro al discharge t.) take place inistead.

There are other features in which a comparison may be made between the auroral light and vacuum discharge. These discharge; when free to arrange themselves in a magnetic field, follow the lines of force; the auroral streamers appear to run parallel to the dipping needle. The colour of such discharges varies with the exhaustion; that of the aurora varies, like that of an air-vacuum, from red almost to white; and in the absence of independent observations to the contrary, we may fairly attribute the variety of tint in the aurora partly to a diversity of elevation, and, consequently, of rarefaction in the region where it takes place, but partly also perbaps to the electrical conditions present anterior to the passage of the di:charse.

These and other features of the phenomenon of the aurora, as well as the kindred subjects of earth currents, the disturbances of the magnetic needle, and the connection of the whole with solar radiation as a predisposing cause, have been brought together under one theoretic view by Prof. Stokes, to whom I am indebted for much of what I have here said on the subject.

Having thus gone throngh, so far as circumstanc s permitted, the experimental and inductive parts of my subject, it might have been very pleasant to have cast aside for a few moment the links which connect strict induction with what may be termed the fixed points of ascertained fact ; and, restrained only by the more elastic bonds of scientific imagination, to have indulged in speculations about things still lying on the borders of science and of dreamland. But I must leave each of you to follow out this vein of thourht after your own fashion; and, confinin myself to a single remark, I will simply indicate the direction in which my own thoughts on the present subject are inclined to turn. The remark is this: If in the search for a solution of the mystery of electricity there be one element more deserving our attention rather than another, it is that of time. We have utilised this element in our experiments with the revolving mirror; and we have touched upon its more subtle influences in our conclusions about the small time quantities in relation to the discharge.

All operations of nature take place in time. It is in the timesequence of phases, often apparently simultaneous, but in reality successive, that we may hope to strike the origin of many complicated phenomena. Time is the ocean beneath whose waves and whose currents the secret fountains of truth are to be sought. Time is the ocean whose mighty stream encircles our life. Time is the ocean whose "countless smiles" gave birth to Venus and the Nereids and all the infinite forms of beauty and of brightness which play around our youth. Time is the ocean from whence sprang also the steeds of Neptune, typical of the strength of our mo e mature years. Time is the ocean in whose loving arms we fall asleep, when the sun sinks low on the horizon, and the shades of night are creeping over the heavens, and all things tell us that our course is run.

\section{BIOLOGY AS AN ACADEMICAL STUDY} II.

I T may help to the understanding of what I mean by a sound method of biological teaching if I give a brief outline of the course of study I hope to pursue with my students this session. It is hardly necessary for me to state that this course is derived from Prof. Huxley's by a natural process of descent with modification.

In the first place there will be some four or five lecture; on a common flowering plant, giving an account of its ordinary structure as seen by the naked eye, of its microscopic structure, of its physiol igy, and of the process of its development. After each lecture the students will examine for themselves the plant described, learning not only to dissect it in the ordinary way, but to make preparations for the microscope. By this means they will be familiarised with the use of the microscope, the employment of stainin $y$ fluids, and other reagents used in the investigation of minute structure, and with the chief processes of manipulation. As the laboratory will be ojen for nine hours a week, so $a ;$ to give three hours for working out what has been described in each hour's lecture, it is to be expected that a student of average intelligence will, by the time this part of the course is over, have a very fair notion of what a flowering plant is, of the procesies by which its life is carried on, and of the manner in which it originates.

The next few lectures, and the corresponding portion of the practical course, will be occupied with a sinilar treatment of an animal: the one selected, as on the whole the most convenient and the most instructive, being the common sea crayfish of our markets. In the examination of this organism, the students will learn something of the art of dissection, and will further apply the knowledge of microscopic structure which the study of the plant has given them, to the far more difficult problems of animal histology. The study of the crayfish, and the comparison of it, point by point, with the plant, should give a clear conception of the main points of difference and of likeness between the more highly organised animals and plants--between aninials and plants as they are generally known to us.

In dealing with these types in the lectures it will be my aim always to proceed from the known to the unknown; to begin with points which every one who has seen a flowering plant or a crayfish mu-t have noticed, gradually leading up to such points of structure as require minute observation to verify them, and above all never to give a definition or a general statement without first supplying the facts from which it is legitimately deducible.

Next, I propose to take a number of types selected on the one hand from the lowest plants, on the other from the lowest animals: to show how these unicellular organisms agree in structure and in the nature of their physiological processes with the individual cells of which the bodies of the higher plants and animals are made up, and to point out how, in denlins with these lowest members of the two kingdoms of organic nature, the boundary line between the two kingdoms tends to disappear, and it becomes very difficu't, sometimes even impossible, to say what is a plant and what an animal. The study of these lowly forms will also lead to the question of the origin of life, and it will be necessary to say something of the attempts which have been made to establish the doctrine of spontaneous generation, and to discuis their value.

The consideration of a few other animal and vegetable types, especially such as, although multicellular, exhibit none of the complex tissues found in the higher animals and plants, will bring the introductory part of the course to a close-the part which deals with the general facts and principles of biology. In it the student should learn how animals and plants agree with and differ from each other, and from inorganic bodies; what are the relations of animals and plants to one another, and to

${ }^{x}$ Inaugural Lecture delivered in the University Library, May 2, $188 \mathrm{x}$, by T. Jeffery Parker, B.Sc., Lond., Professor of Biology in the University of Otago. Continued from p. 546. 